\title{
Sensorless Direct Torque Control of PMSM using Unsected Kalman Filter *
}

\author{
Zhen Chen* Liang Wang* Xiangdong Liu* \\ * School of Automation, Beijing Institute of Technology, Beijing \\ 100081, P.R.China \\ (e-mail: $\{$ chenzhen76, nicholas1233, xdliu\}@ bit.edu.cn).
}

\begin{abstract}
This paper considers the sensorless direct torque control of a Permanent Magnet Synchronous Motor (PMSM) by using Unscented Kalman Filtering (UKF) to estimate the flux-linkage, the rotor speed and the position. UKF adapts the unscented transformation (UT) algorithm and symmetrical sampling strategy. The UKF algorithm represents a serious strongpoint to the Extended Kalman Filter (EKF) for the highly nonlinear systems. UKF ensures high estimation precision and simple calculation. The sensorless Direct Torque Control (DTC) for PMSM is designed in this paper. The estimating errors when using UKF and EKF are compared. The effectiveness of this method is verified through several simulations. The DTC based on UKF for PMSM has good speed and torque response performances. This method has rapid astringency of estimated error.
\end{abstract}

Keywords: Direct Torque Control, PMSM, Unscented Kalman Filtering, Flux Linkage Estimation, Sensorless.

\section{INTRODUCTION}

PMSM is becoming a hot research topic in the field of electricity transmission, due to its various advantages such as high power density, high efficiency, high reliability, and wide implementation in many occasions requiring high performance. As a high performance control scheme, DTC has been widely used in induction motor drives, also has witnessed many research results in PMSM. In many circumstances, both the rotor speed and position use encodes or sensors, whose performance is proportional to the cost. Therefore, using low-performance sensors and encodes inevitably cause system noise and poor precision, while improving the cost of devices may make the reliability and robustness become worse. There are also harsh environments where the position and velocity encoders can not be simply installed. One typical example is the single gimbal control moment gyro servo drive system, which requires high reliability and low speed stability. Utilizing a high-precision encoder will certainly increase the cost, and decrease reliability for the harsh environments in space. Therefore, sensorless motor control has drawn attention from many research scholars.

So far, the research of sensorless technology mainly concentrates on estimating the position and velocity, specifically for an AC servo motor, on the estimation of the position and speed estimation of the vector control system. For the DTC system, several scholars have proposed various sensorless control strategies by using different estimation methods (Lascu et al. (2004); Feng and Xu (2006); Zhang et al. (2007); Huang et al. (2006)). EKF was used widely in these methods. Borsje et al. (2005) and Nanga-Ndjana

\footnotetext{
^ This paper is supported by National Natural Science Foundation of China (Grant No. 10872030).
}

and Lautier (2006) developed EKF and UKF algorithms for the drives modes of PMSM-SVPWM. However, to the knowledge of the authors, utilizing UKF to estimate PMSM-DTC flux hasnt been yet studied. Despite the wide applications of EKF, there are some shortcomings of EKF, e.g, the high-order terms neglected while using Taylor expansion to linearize a nonlinear function may lead to a fairly large estimation error, and sometimes cause the filter unstable. Besides, the Jacobian matrix needs to be calculated at each control period, which becomes more difficult as the dimension of the system is higher.

In order to tackle the disadvantages of using EKF to deal with the nonlinear filtering problems, Julier et al. (1995) proposed UKF method in 1995 for the first time. Wan and VanDer-Merwe (2000) and VanDer-Merwe and Wan (2001) further studied UKF. Pan et al. (2005) pointed out the following advantages of UKF over EKF:

(1) approximate the probability density function of nonlinear distribution, instead of the nonlinear function itself.

(2) the calculation accuracy of nonlinear distribution statistics is up to at least 2 order. Even higher-order accuracy can be achieved if special sampling strategies are adapted, e.g., 4-order sampling of gaussian distribution and sample skewness.

(3) it does not need to compute the Jacobian matrix, and moreover, it can handle non-additive noises and discrete systems, thereby extending the application area.

(4) the calculation amount is of the same order with EKF.

(5) it avoids the particle recession problem, for it uses the deterministic sampling approach, rather than the random sampling of ions filters. 
This paper uses UKF to estimate the flux-linkage, rotor speed and position, and further proposes a sensorless direct torque control system of PMSM. Therefore, the study of this paper has significant potential practice value.

\section{THE PRINCIPLE OF UKF ESTIMATOR}

As UKF approximates the probability distribution of a nonlinear function rather than approximates the linear function itself, it uses a deterministic sampling approach to capture the mean and covariance estimates with a minimal set of sample points. In order to avoid the linearization error of EKF, UKF uses a more accurate approximation of UT, which, based on the prior state of input, uses deterministic sampling approach to capture the mean and covariance, and to calculate the point set of Sigma, then makes of nonlinear transformation of each Sigma point. Julier et al. (2000) discussed the involved sampling strategies. This paper adapts the symmetrical sampling strategy, taking into consideration the calculation amount and the realization problem.

Consider the following non-linear system:

$$
\begin{aligned}
& X_{k+1}=F_{k}\left(X_{k}, U_{k}\right)+w_{k} \\
& y_{k+1}=H_{k}\left(X_{k+1}\right)+v_{k}
\end{aligned}
$$

where $X_{k}$ is the L-dimensional state vector of the system, $F_{k}\left(X_{k}, U_{k}\right)$ is the state propagation equation, $U_{k}$ is the control input vector, $w_{k}$ is the process noise, which is assumed to be the zero-mean Gaussian white noise with $Q_{k}$ as its variance (denoted by $w_{k} \sim N\left(0, Q_{k}\right)$ ), $y_{k}$ is the measurement value, $H_{k}\left(X_{k}\right)$ is the measurement equation, and $v_{k}$ is the measurement noise, which also satisfies $v_{k} \sim N\left(0, R_{k}\right)$. Suppose that $X_{0}$ and $P_{0}$ are, respectively, the initial value and variance of the system's state vector.

The UKF algorithm mainly consists of three steps:

(1) the sampling and calculation of Sigma points.

(2) time update (the prediction step).

(3) measurement update (estimation correction ).

Specifically,

\section{Step 1 Initialization, computing Sigma points and choosing weights}

The initial states and the covariance matrices are as follows:

$$
\begin{aligned}
& \hat{X}_{0}=E\left[X_{0}\right] \\
& P_{0}=\operatorname{chol}\left\{E\left(X_{0}-\hat{X}_{0}\right)\left(X_{0}-\hat{X}_{0}\right)^{T}\right\}
\end{aligned}
$$

According to the optimal state estimate $\hat{X}_{k-1}$ of time $k-1$, we can obtain the $2 L+1$ sets of Sigma points as $\left\{x_{i, k \mid k-1}\right\}$, by using the symmetrical sampling approach. the set of Sigma points are computed as follows:

$$
\left\{\begin{array}{l}
\chi_{0, k \mid k-1}=\hat{X}_{k-1} \\
\chi_{i, k \mid k-1}=\hat{X}_{k-1}+\left(\sqrt{(L+\lambda) P_{k-1}}\right)_{i} \\
\chi_{i+L, k \mid k-1}=\hat{X}_{k-1}-\left(\sqrt{(L+\lambda) P_{k-1}}\right)_{i}
\end{array}\right.
$$

with $i=1,2, \cdots, L$.

The coefficients of the mean and covariance are weighted as:

$$
\left\{\begin{array}{l}
W_{0}^{(m)}=\frac{\lambda}{L+\lambda} \\
W_{0}^{(c)}=\frac{\lambda}{L+\lambda}+1-\alpha^{2}+\beta \\
W_{i}^{(m)}=W_{i}^{(c)}=\frac{1}{2(L+\lambda)}
\end{array}\right.
$$

where the superscript $(m)$ and $(c)$ denote, respectively, the weight for the mean and covariance, $\lambda$ is the proportional parameter, used to adjust the distance between Sigma points and $\hat{X}$, which is taken as $\lambda=\alpha^{2}(L+\kappa)-L$, the constant $\alpha$ determines the spread of Sigma points, usually is chosen to be a small positive value (e.g., $10^{-4} \leq$ $\alpha \leq 1), \kappa$ is a constant, usually being either 0 or $3-\bar{L}$, $\beta$ is a parameter related to the prior knowledge of the distribution of state $(\beta=2$ is the optimal, when the state is the Gaussian distribution), $\left(\sqrt{(L+\lambda) P_{k}}\right)_{i}$ is the $i-$ th row or column of the square root of $(L+\lambda) P_{k}$, and $W_{i}$ is the weight associated with the $i$-th Sigma point.

Based on the above discussions, (3) and (4) can be rewritten as:

$$
\left\{\begin{array}{l}
\chi_{0, k \mid k-1}=\hat{X}_{k-1} \\
\chi_{i, k \mid k-1}=\hat{X}_{k-1}+\left(\alpha \sqrt{(L+\kappa) P_{k-1}}\right)_{i} \\
\chi_{i+L, k \mid k-1}=\hat{X}_{k-1}-\left(\alpha \sqrt{(L+\kappa) P_{k-1}}\right)_{i}
\end{array}\right.
$$

and

$$
\left\{\begin{array}{l}
W_{0}^{(m)}=1-\frac{L}{\alpha^{2}(L+\kappa)} \\
W_{0}^{(c)}=1-\frac{L}{\alpha^{2}(L+\kappa)}+1-\alpha^{2}+\beta \\
W_{i}^{(m)}=W_{i}^{(c)}=\frac{1}{2 \alpha^{2}(L+\kappa)}
\end{array}\right.
$$

\section{Step 2 Time update (the prediction step)}

The $L \times(2 L+1)$ matrix $\chi_{k-1}$ of Sigma points is constituted by using the $2 L+1$ sets of Sigma points $\left\{\chi_{i, k \mid k-1}\right\}$. In light of the first (1), we can get prediction matrix $\chi_{k \mid k-1}$ by doing a non-linear transformation for each sample point as:

$$
\chi_{i, k \mid k-1}=F_{d}\left(\chi_{i, k-1}, U_{k-1}\right), i=0, \cdots,(2 L+1)
$$

Based on the weights, the mean and covariance matrix of the state are predicted as follows:

$$
\hat{X}_{k \mid k-1}=\sum_{i=0}^{2 L} W_{i}^{(m)} \chi_{i, k \mid k-1}
$$

$\hat{P}_{k \mid k-1}=$

$$
\sum_{\substack{i=0 \\+Q}}^{2 L} W_{i}^{(c)}\left[\chi_{i, k \mid k-1}-\hat{X}_{k \mid k-1}\right]\left[\chi_{i, k \mid k-1}-\hat{X}_{k \mid k-1}\right]^{\mathrm{T}}
$$

According to the second equation of (1), the value of output measurement can be obtained by calculating each of prediction points $\chi_{i, k \mid k-1}$ :

$$
y_{i, k \mid k-1}=H_{k}\left(\chi_{i, k \mid k-1}\right), i=0, \cdots,(2 L+1)
$$

According to the weight of the mean coefficient, the output measurement update is:

$$
\hat{y}_{k \mid k-1}=\sum_{i=0}^{2 L} W_{i}^{(m)} y_{i, k \mid k-1}
$$




\section{Step 3 Measurement update}

Compute the error variance matrix of the update measurement as:

$$
\begin{aligned}
& P_{y_{k} y_{k}}= \\
& \sum_{i=0}^{2 L} W_{i}^{(c)}\left[y_{i, k \mid k-1}-\hat{y}_{k \mid k-1}\right]\left[y_{i, k \mid k-1}-\hat{y}_{k \mid k-1}\right]^{\mathrm{T}}+R
\end{aligned}
$$

Then, calculate the error variance matrix between predicted states and update measurement:

$$
\begin{aligned}
& P_{X_{k} y_{k}}= \\
& \sum_{i=0}^{2 L} W_{i}^{(c)}\left[\chi_{i, k \mid k-1}-\hat{X}_{k \mid k-1}\right]\left[y_{i, k \mid k-1}-\hat{y}_{k \mid k-1}\right]^{\mathrm{T}}
\end{aligned}
$$

Further, compute the Kalman gain:

$$
K_{k}=P_{X_{k} y_{k}} P_{y_{k} y_{k}}^{-1}
$$

Finally, compute the optimal estimation of the state vector and the covariance matrix of time $k$ :

$$
\begin{gathered}
\hat{X}_{k}=\hat{X}_{k \mid k-1}+K_{k}\left(y_{k}-\hat{y}_{k \mid k-1}\right) \\
P_{k}=P_{k \mid k-1}-K_{k} P_{y_{k} y_{k}} K_{k}^{-1}
\end{gathered}
$$

The detailed realization of UKF will be discussed, by combining with the state estimation of PMSM-DTC system.

\section{THE MODEL OF PMSM-DTC}

In PMSM-DTC system, the amplitude of flux linkage and electromagnetic torque are primarily to be controlled. The PMSM voltage and flux linkage equations in the two-axis stationary $\alpha \beta$ reference frame are:

$$
\begin{gathered}
\left\{\begin{array}{c}
u_{s \alpha}=\frac{d \psi_{s \alpha}}{d t}+R_{s} i_{s \alpha} \\
u_{s \beta}=\frac{d \psi_{s \beta}}{d t}+R_{s} i_{s \beta}
\end{array}\right. \\
\left\{\begin{array}{l}
\psi_{s \alpha}=L_{s} i_{s \alpha}+\psi_{f} \cos \theta_{r} \\
\psi_{s \beta}=L_{s} i_{s \beta}+\psi_{f} \sin \theta_{r}
\end{array}\right.
\end{gathered}
$$

where $u_{s \alpha}, u_{s \beta}, i_{s \alpha}, i_{s \beta}$ are the voltages and currents on the $\alpha$ and $\beta$ axis, respectively, $\psi_{s \alpha}, \psi_{s \beta}$ are the stator flux linkage on the $\alpha$ and $\beta, \psi_{\mathrm{f}}$ is the magnet flux linkage, $L_{s}, R_{s}$ are the stator inductance and resistance, respectively, and $\theta_{r}$ is the relative position between stator flux and rotor flux, which is called the rotor electrical angle.

Substituting (18) into (17) gives:

$$
\left\{\begin{array}{l}
\frac{d \psi_{s \alpha}}{d t}=-\frac{R_{s}}{L_{s}} \psi_{s \alpha}+\frac{R_{s}}{L_{s}} \psi_{f} \cos \theta_{r}+u_{s \alpha} \\
\frac{d \psi_{s \beta}}{d t}=-\frac{R_{s}}{L_{s}} \psi_{s \beta}+\frac{R_{s}}{L_{s}} \psi_{f} \sin \theta_{r}+u_{s \beta}
\end{array}\right.
$$

where $L_{s}$ is the stator inductance of PMSM, $R_{s}$ is the stator resistance.

In order to design the UKF observer, the angular velocity $\omega_{e}$ is regarded as constant within the sampling period $T_{s}$, and $\omega_{e}$ is the changing rate of angle $\theta_{r}$, i.e.,

$$
\frac{d \omega_{e}}{d t}=0, \frac{d \theta_{r}}{d t}=\omega_{e}
$$

The UKF estimator is independent of the motor mechanical parameters, when using $d \omega_{e} / d t=0$ as the dynamic equation of rotor speed, thereby overcoming the impact of the uncertainties of the mechanical parameters on estimator. Here, the mechanical parameters include load torque disturbance, viscous friction coefficient, rotor inertia, and so forth.

Then, we get the nonlinear mathematical model of PMSM as:

$$
\begin{gathered}
\dot{X}=f(X)+B U \\
y=h(X)
\end{gathered}
$$

where $X=\left[\begin{array}{llll}\psi_{s \alpha} & \psi_{s \beta} & \omega_{e} & \theta_{e}\end{array}\right]^{\mathrm{T}}$ is the state variable, $U=$ $\left[\begin{array}{lll}u_{s \alpha} & u_{s \beta}\end{array}\right]^{\mathrm{T}}$ is the control input, and $y=\left[\begin{array}{ll}i_{s \alpha} & i_{s \beta}\end{array}\right]^{\mathrm{T}}$ is the output vector. In the PMSM-DTC system, current and voltage sensors measure the three-phase current and voltage, so the stator current $i_{s \alpha}, i_{s \beta}$ and voltage $u_{s \alpha}, u_{s \beta}$ can be obtained through transformation between threeaxis stationary $\mathrm{ABC}$ coordinate and two-axis stationary $\alpha \beta$ coordinate. The system state matrices are defined as:

$$
\begin{gathered}
f(X)=\left[\begin{array}{c}
-\frac{R_{s}}{L_{s}} \psi_{s \alpha}+\frac{R_{s}}{L_{s}} \psi_{f} \cos \theta_{e} \\
-\frac{R_{s}}{L_{s}} \psi_{s \beta}+\frac{R_{s}}{L_{s}} \psi_{f} \sin \theta_{e} \\
0
\end{array}\right], B=\left[\begin{array}{ll}
1 & 0 \\
0 & 1 \\
0 & 0 \\
0 & 0
\end{array}\right] \\
h(X)=\left[\begin{array}{c}
\frac{1}{\omega_{s}}\left(\psi_{s \alpha}-\psi_{f} \cos \theta_{e}\right) \\
\frac{1}{L_{s}}\left(\psi_{s \beta}-\psi_{f} \sin \theta_{e}\right)
\end{array}\right]
\end{gathered}
$$

Equation (20) can be is expressed in the discrete dynamic equation form of UKF estimator.

$$
\begin{gathered}
X_{k+1}=F_{k}\left(X_{k}\right)+B_{k} U_{k}+w_{k} \\
y_{k}=H_{k}\left(X_{k}\right)+v_{k}
\end{gathered}
$$

where

$$
\begin{aligned}
& F_{k}\left(X_{k}\right)=X_{k}+f\left(X_{k}\right) T_{s} \\
& B_{k}=B T_{s} \\
& H_{k}\left(X_{k}\right)=h\left(X_{k}\right)
\end{aligned}
$$

$w_{k}$ and $v_{k}$ are the noise and measurement noise, respectively, which are independent zero-mean Gaussian process, $T_{s}$ is the sampling time, and the expressions of $F_{k}\left(X_{k}\right)$ and $H_{k}\left(X_{k}\right)$ are as follows:

$$
\begin{gathered}
F_{k}\left(X_{k}\right)=\left[\begin{array}{c}
\left(1-\frac{R_{s}}{L_{s}} T_{s}\right) \psi_{s \alpha}+\frac{R_{s}}{L_{s}} \psi_{f} T_{s} \cos \theta_{r} \\
\left(1-\frac{R_{s}}{L_{s}} T_{s}\right) \psi_{s \beta}-\frac{R_{s}}{L_{s}} \psi_{f} T_{s} \sin \theta_{r} \\
\omega_{e} \\
\theta_{r}+\omega_{e} T_{s}
\end{array}\right]_{X=X_{k}} \\
H_{k}\left(X_{k}\right)=h\left(X_{k}\right)=\left[\begin{array}{c}
\frac{1}{L_{s}}\left(\psi_{s \alpha}-\psi_{f} \cos \theta_{e}\right) \\
\frac{1}{L_{s}}\left(\psi_{s \beta}-\psi_{f} \sin \theta_{e}\right)
\end{array}\right]_{X=X_{k}}
\end{gathered}
$$

\section{PMSM-DTC SYSTEM BASED ON UKF}

The ectromagnetic torque $T_{e}$ of PMSM is:

$$
T_{e}=\frac{3 P_{m}}{2 L_{s}} \psi_{f}\left(\psi_{s \beta} \cos \theta_{r}-\psi_{s \alpha} \sin \theta_{r}\right)
$$


The calculation formula for the square of stator flux amplitude is:

$$
\left|\psi_{\mathrm{s}}\right|^{2}=\left(\psi_{s \alpha}\right)^{2}+\left(\psi_{s \beta}\right)^{2}
$$

According to the UKF algorithm and the dynamic model of PMSM-DTC, the flux-linkage, rotor speed and position of PMSM-DTC system are estimated. Then according to (24) and (25), PMSM-DTC of torque and flux-linkage amplitude closed-loop system are established through calculating the feedbacks of DTC. The block diagram of the PMSM-DTC based on UKF is shown as Fig.1.

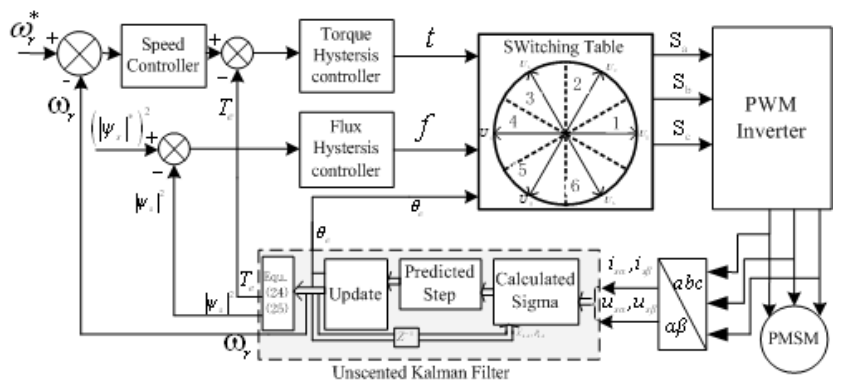

Fig. 1. Diagram of the PMSM DTC based on UKF

The direct torque control strategy is that the deviation between reference speed and the estimated speed of UKF through speed controller, then the electromagnetic torque $T_{e}^{*}$ is obtained, and then $\Delta T_{e}$ is known by subtracting the estimated electromagnetic torque $T_{e}$ of UKF, $\Delta \Psi_{s}$ is obtained through reference flux-linkage and the estimated flux-linkage. The torque deviation and the amplitude deviation of flux-linkage through the controller, and two outputs $t$ and $f$ are derived, through combining with the interaction of estimated rotor position of UKF to select the appropriate voltage vector, and finally PMSM is derived through the voltage source inverter.

\section{SIMULATION AND EXPERIMENT RESULTS}

According to the PMSM-DTC system and UKF algorithm, one will verify the state estimation performance of UKF. Here, the parameters of PMSM are given in table.1. System sampling period is $50 \mu$ s and the initial speed is $1500 \mathrm{r} / \mathrm{min}$. Then the speed is changed to $700 \mathrm{r} / \mathrm{min}$ at $0.1 \mathrm{~s}$, and further changed to $1000 \mathrm{r} / \mathrm{min}$ at $0.2 \mathrm{~s}$. The experimental platform is shown in Fig.2. and the simulation results are displayed in Fig.3-Fig.9.

Table 1. The parameters of PMSM

\begin{tabular}{cc} 
Parameter name & Parameter value \\
\hline Stator resistance $\left(R_{s}\right)$ & $0.93 \Omega$ \\
Rotor inertia $(J)$ & $5 \times 10^{-4} \mathrm{Kg} \cdot \mathrm{m}^{2}$ \\
Stator inductance $\left(L_{s}\right)$ & $0.27 \mathrm{mH}$ \\
Permanent magnet flux $\left(\Psi_{f}\right)$ & $0.01 \mathrm{~Wb}$ \\
Pole pairs $\left(P_{m}\right)$ & 4 \\
Bus voltage $\left(U_{d c}\right)$ & $15 \mathrm{~V}$ \\
\hline
\end{tabular}

The estimated speed of UKF and the actual speed are compared in Fig.3. Fig.4 is the error curve between the estimated speed of UKF and the actual speed, which shows high tracking accuracy. The relative average error is about $1.93 \%$ when the speed is changing, then it turns to be about $0.13 \%$ in the steady state. Fig. 5 shows the rotor position estimation of UKF. Fig.6 shows the error curve of estimated position, from which can easily be observed that the position estimation error is slightly larger when the speed is changing state than it is steady. Fig.7 shows the estimated stator flux-linkage of UKF. Fig.8 shows the error curve of the estimated stator flux-linkage, in which $\Delta \Psi_{s \alpha}, \Delta \Psi_{s \beta}$ are, respectively, the error curve of the estimated stator flux-linkage on the $\alpha$ and $\beta$ axis. When adding $0.05 \mathrm{~N} . \mathrm{m}$ load disturbance at $0.25 \mathrm{~s}$, the estimated electromagnetic torque curve is shown as Fig.9, which shows that PMSM-DTC has fast torque response, and the disturbance is reflected only when the electromagnetic torque and the stator current change. It can be seen from Fig.3 that the speed adjusts to the given value rapidly at $0.25 \mathrm{~s}$, which improves the smooth of speed and the disturbance rejection capability for the direct torque control of servo system.

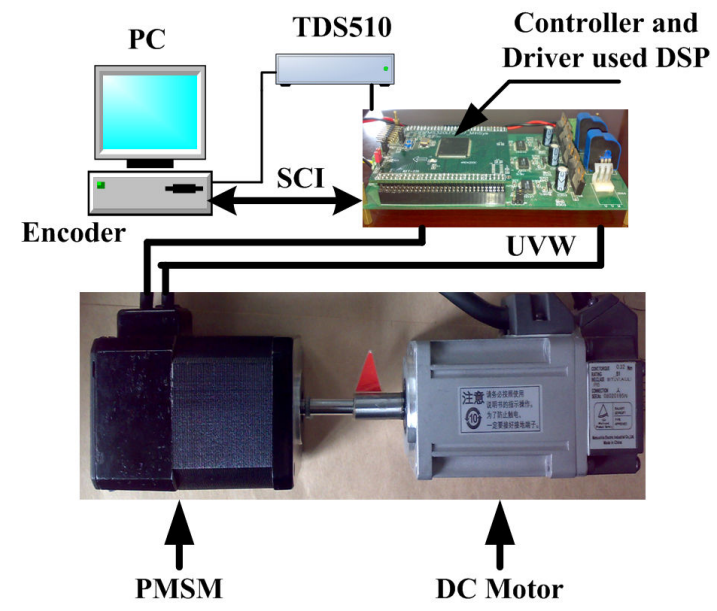

Fig. 2. Experimental platform of PMSM-DTC

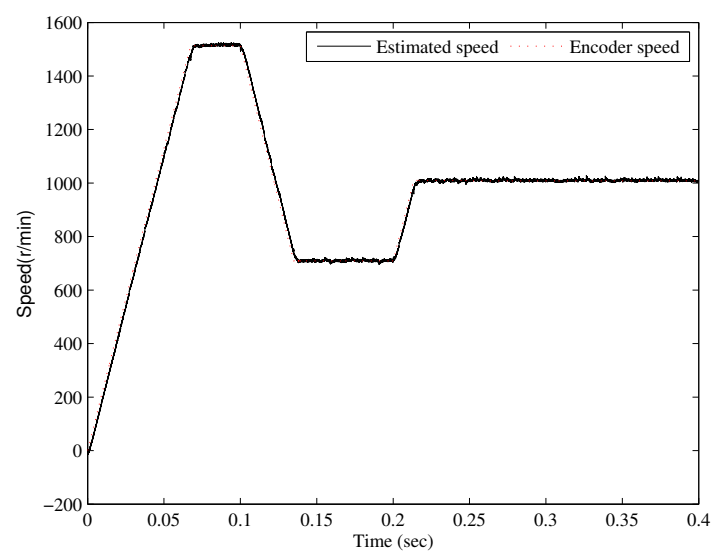

Fig. 3. Estimated speed by UKF

As analyzed in Julier et al. (2000), when EKF is used to deal with the filtering problem of nonlinear systems, the nonlinear function is linearized by Taylor expansion which neglects the higher-order terms, thus it has only first-order accuracy. By contrast, UKF approximates the probability density function of the nonlinear function, rather than the nonlinear function itself. As pointed out in Julier et al. (2000), the calculation accuracy of nonlinear distribution 


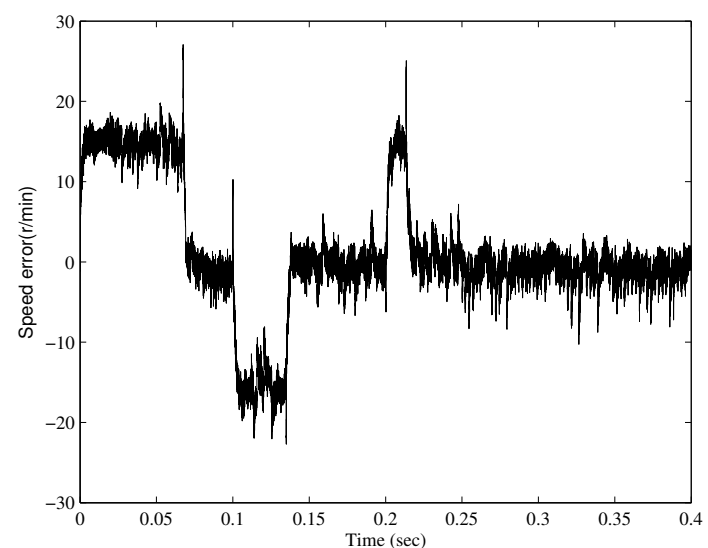

Fig. 4. Estimated speed error by UKF

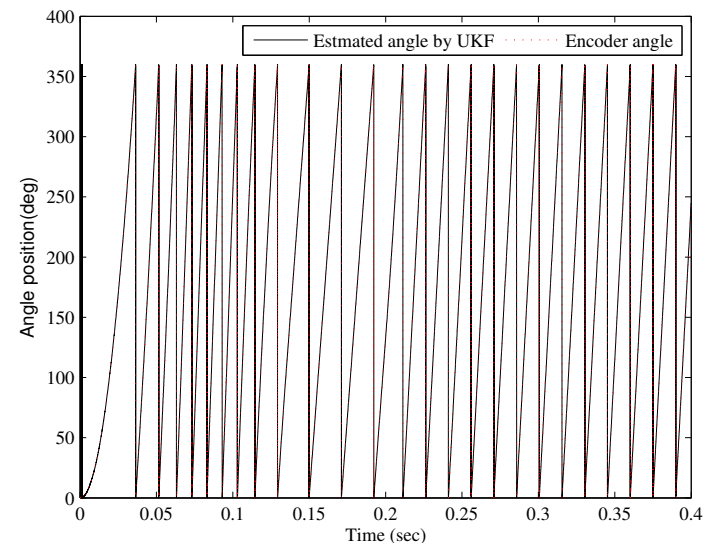

Fig. 5. Estimated position by UKF

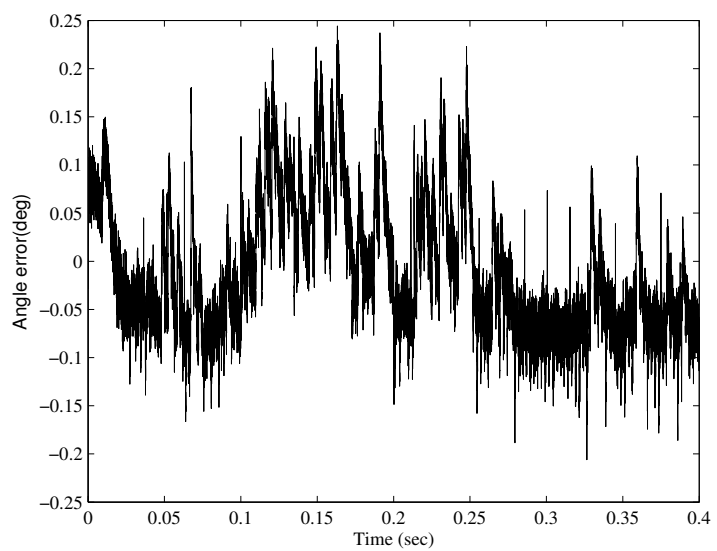

Fig. 6. Estimated position error by UKF

statistics is at least 2-order. This paper adopts symmetric sampling for the Sigma points to ensure the approximation accuracy of any distribution reaches 2-order of Taylor expansion, therefore, UKF gives more accurate estimation than EKF. The experimental results are shown in Fig.10Fig.12.

Fig.10 compares the filter prediction error when using UKF and EKF to estimate the speed. The estimation

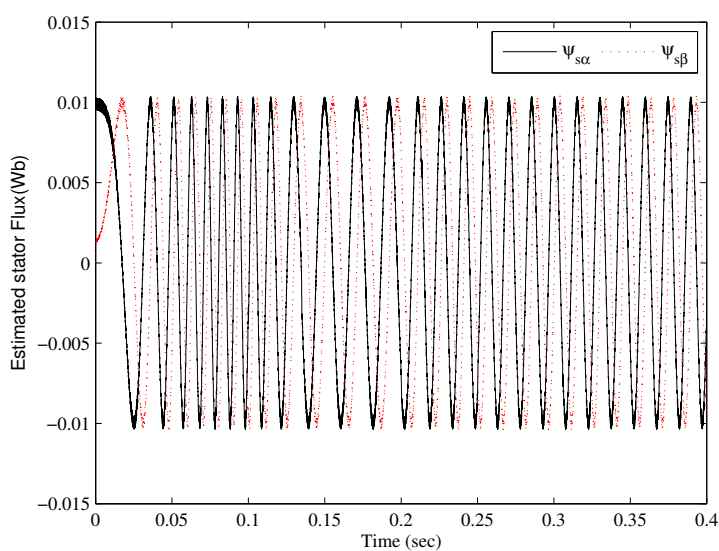

Fig. 7. Estimated flux linkage of stator by UKF

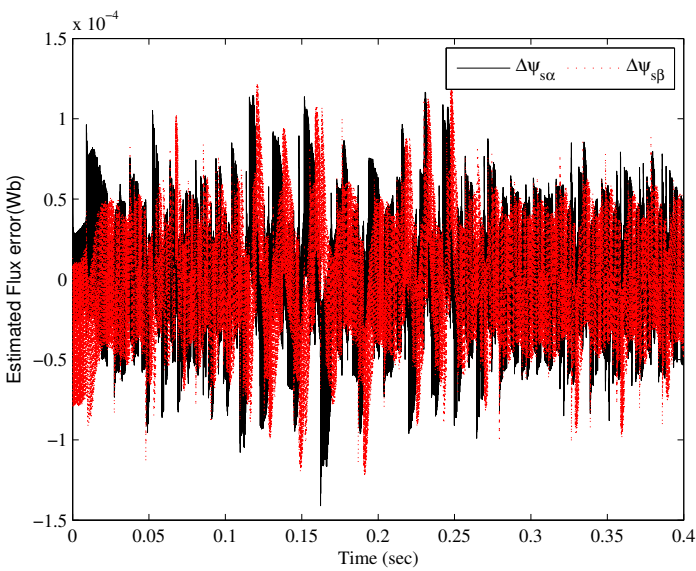

Fig. 8. Estimated flux linkage error of stator by UKF

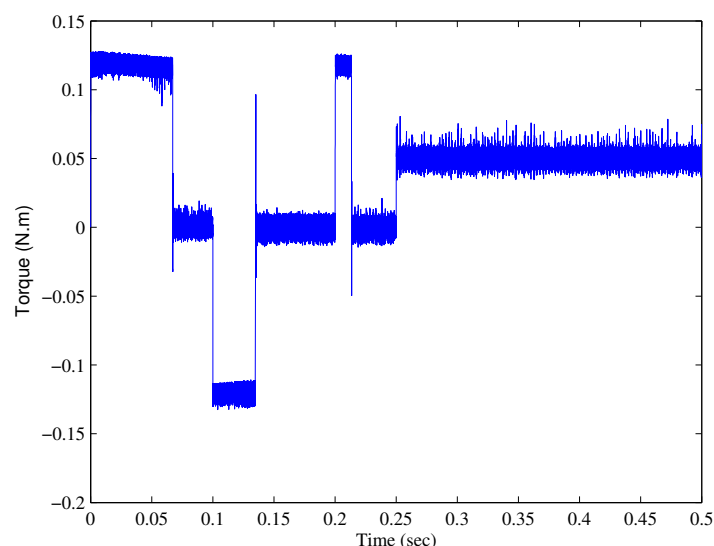

Fig. 9. Torque curve adding load disturbance at $0.2 \mathrm{~s}$

accuracy of UKF is higher than that of EKF. The relative mean of the EKF estimated speed error is about $3.27 \%$, while that of UKF is $1.93 \%$. In the steady state, the estimation of UKF is more accurate with small fluctuations in speed, especially when the sudden change of speed caused by the load disturbance at $0.25 \mathrm{~s}$, the estimation accuracy of UKF is 1.9 times than that of EKF, so UKF has both good dynamic and static performances. Fig.11 shows the error curves when using respectively UKF and 


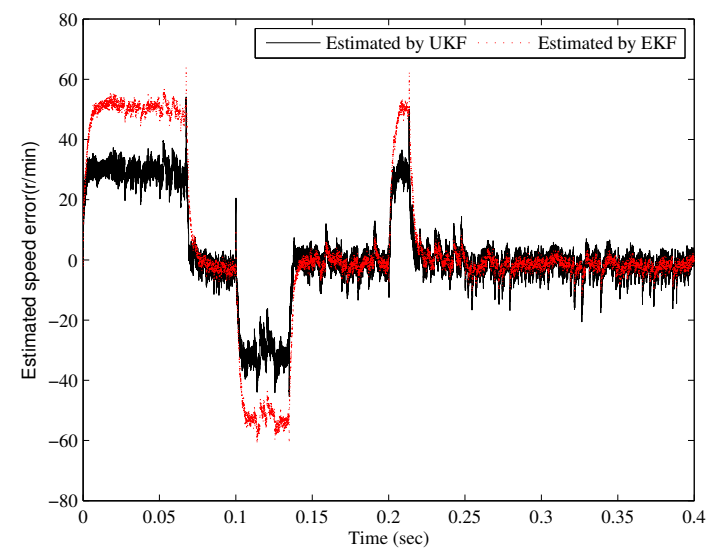

Fig. 10. Error curves of estimated speed by UKF and EKF

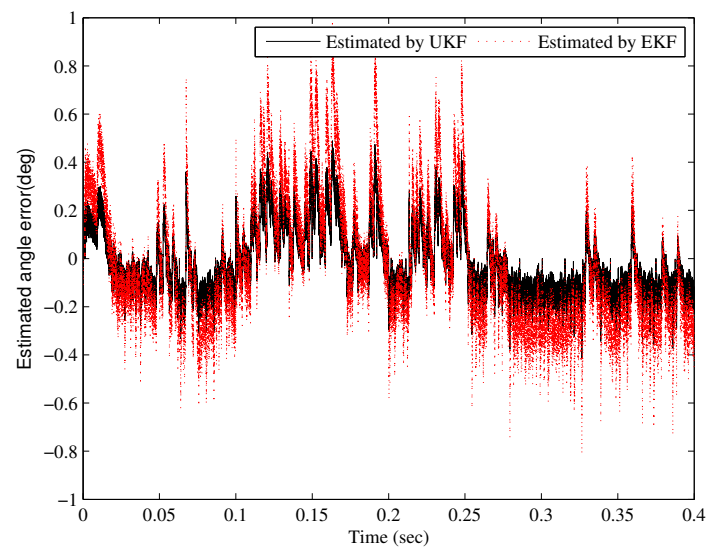

Fig. 11. Prediction error of estimated position by UKF and $\mathrm{EKF}$

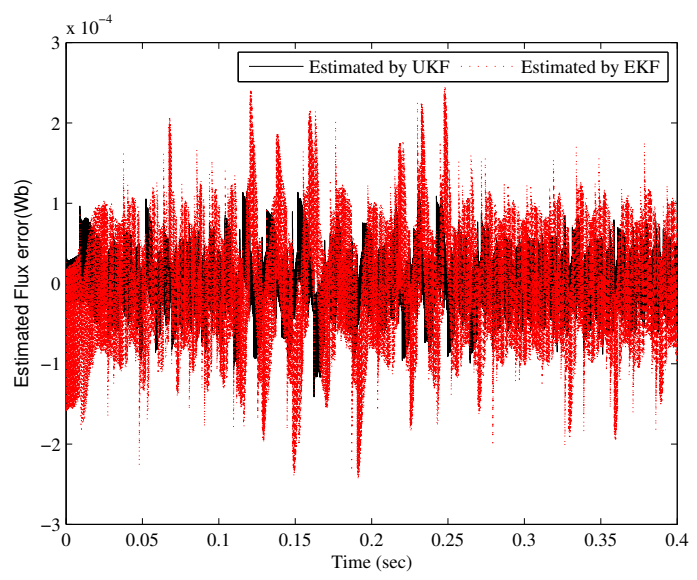

Fig. 12. Error curves of estimated flux-linkage by UKF and $\mathrm{EKF}$

EKF to estimate the position and Fig.12 depicts the error curves when using respectively UKF and EKF to estimate the flux linkage.

\section{CONCLUSIONS}

This paper presents a sensorless direct torque control of a PMSM using UKF for flux-linkage, rotor speed and position estimation. Comparison with EKF is done. As seen from the experiment and simulation results, the effectiveness of UKF for the state estimation of PMSMDTC is verified. Meanwhile, it shows that the PMSMDTC system based on UKF has a quick dynamic response and a satisfactory steady-state performance. Therefore, the outstanding performance of UKF in sensorless control is illustrated.

\section{REFERENCES}

Borsje, P., Chan, T., Wong, Y., and Ho, S. (2005). A comparative study of kalman filtering for sensorless control of a permanent-magnet synchronous motor drive. In 2005 IEEE International Conference on Electric Machines and Drives, 815-822. IEEE, San Antonio, TX.

Feng, J. and $\mathrm{Xu}$, J. (2006). Permanent magnet synchronous machines direct torque control system based on adaptive stator flux observer. Proceedings of the CSEE, 26, 122-127.

Huang, M., Moses, A., and Anayi, F. (2006). The comparison of sensorless estimation techniques for pmsm between extended kalman filter and flux-linkage observer. In Twenty-First Annual IEEE Applied Power Electronics Conference and Exposition, 654-660. IEEE, Dallas, Texas.

Julier, S., Uhlmann, J., and Durrant-Whyte, H. (1995). A new approach for filtering nonlinear systems. In Proceedings of the American Control Conference, volume 3, 1628-1632. IEEE, Seattle, Washington.

Julier, S., Uhlmann, J., and Durrant-Whyte, H. (2000). A new approach for the nonlinear transformat ion of means and covariances in filters and estimators. IEEE Transactions on Automatic Control, 45, 477-482.

Lascu, C., Boldea, I., and Blaabjerg, F. (2004). Direct torque control of sensorless induction motor drives: a sliding-mode approach. IEEE Transactions on Industry Applications, 40, 582-590.

Nanga-Ndjana, H. and Lautier, P. (2006). Sensorless vector control of an ipmsm using unscented kalman filtering. In IEEE International Symposium on Industrial Electronics, volume 3, 2242-2247. IEEE, Montreal, Que.

Pan, Q., Feng, Y., and Ye, L. (2005). Survey of a kind of nonlinear filters-ukf. Control and Decision, 20, 481-489.

VanDer-Merwe, R. and Wan, E. (2001). The square-root unscented kalman filter for state and parameter estimation. In IEEE International Conference on Acoustics, Speech, and Signal Processing, 3461-3466. IEEE, Salt Lake City.

Wan, E. and VanDer-Merwe, R. (2000). The unscented kalman filter for nonlinear estimation. In IEEE Proc. of the Symposium 2000 on Adaptive System for Signal Processing, Communication and Control, 153-158. IEEE, Lake Louis, Alberta, Canada.

Zhang, M., Xiao, X., and Li, Y. (2007). Speed and flux linkage observer for permanent magnet synchronous motor based on ekf. Proceedings of the CSEE, 27, 36-40. 